\title{
An Eneström-Kakeya theorem for new classes of polynomials
}

\author{
William "TY" Frazier AND Robert GARDNER
}

\begin{abstract}
Consider the class of polynomials $P(z)=\sum_{j=0}^{n} a_{j} z^{j}$ with $0 \leq a_{0} \leq a_{1} \leq \cdots \leq a_{n}$. The classical Eneström-Kakeya Theorem states that any polynomial in this class has all its zeros in the unit disk $|z| \leq 1$ in the complex plane. We introduce new classes of polynomials by imposing a monotonicity-type condition on the coefficients with all indices congruent modulo $m$ for some given $m \leq n$. We give the inner and outer radii of an annulus containing all zeros of such polynomials. We also give an upper bound on the number of zeros in a disk for polynomials in these classes.
\end{abstract}

\section{Introduction}

A classic result concerning the location of the zeros of a polynomial of a complex variable is the so-called Eneström-Kakeya Theorem.

Theorem 1.1 (Eneström-Kakeya). If $P(z)=\sum_{j=0}^{n} a_{j} z^{j}$ is a polynomial of degree $n$ with coefficients satisfying $0 \leq a_{0} \leq a_{1} \leq \cdots \leq a_{n}$, then all the zeros of $P$ lie in $|z| \leq 1$.

In 1893, Gustav Eneström published Theorem 1.1 in Swedish in a paper on the theory of pension funds [4]. Soichi Kakeya [12] independently published a slightly more general result in 1912. Eneström published a French translation of his 1893 proof in 1920 (see [5]). Theorem 1.1 has thus become known as the Eneström-Kakeya Theorem. For more details on the history of this result (and a survey of its generalizations) see [8].

An early and elegant generalization of the Eneström-Kakeya Theorem is Theorem 1.2, due to Joyal et al. [11].

Received August 15, 2018.

2010 Mathematics Subject Classification. 30A10.

Key words and phrases. Location of zeros of polynomials; Eneström-Kakeya theorem. http://dx.doi.org/10.12697/ACUTM.2019.23.11 
Theorem 1.2. If $P(z)=\sum_{i=0}^{n} a_{j} z^{j}$ is a polynomial of degree $n$ with coefficients satisfying $a_{0} \leq a_{1} \leq \cdots \leq a_{n}$, then all the zeros of $P$ lie in $|z| \leq\left(a_{n}-a_{0}+\left|a_{0}\right|\right) /\left|a_{n}\right|$.

Aziz and Mohammad [1] gave a result related to the Eneström-Kakeya Theorem, but concerning analytic functions and a " $t$ condition" on the real and imaginary parts of the coefficients. We state their result as Theorem 1.3 .

Theorem 1.3. Let $f(z)=\sum_{j=0}^{\infty} a_{j} z^{j}$ be analytic in $|z| \leq t$. If $\operatorname{Re}\left(a_{j}\right)=$ $\alpha_{j}$ and $\operatorname{Im}\left(a_{j}\right)=\beta_{j}$ for $j=0,1,2, \ldots$ and for some $k$ and $r$,

$$
\begin{gathered}
\alpha_{0} \leq t \alpha_{2} \leq \cdots \leq t^{k} \alpha_{k} \geq t^{k+1} \alpha_{k+1} \geq \cdots, \text { and } \\
\beta_{0} \leq t \beta_{2} \leq \cdots \leq t^{r} \beta_{r} \geq t^{r+1} \beta_{r+1} \geq \cdots,
\end{gathered}
$$

then $f$ has all its zeros in

$$
|z| \geq \frac{t\left|a_{0}\right|}{2\left(\alpha_{k} t^{k}+\beta_{r} t^{r}\right)-\left(\alpha_{0}+\beta_{0}\right)} .
$$

Gardner and Govil [6, 7] put hypotheses similar to those of Theorem 1.3 on the coefficients of polynomials and gave a number of generalizations of the Eneström-Kakeya Theorem. Aziz and Zargar [2] introduced the idea of imposing a $t$ condition on the even indexed and the odd indexed real coefficients (separately) of a polynomial. Cao and Gardner [3] applied such a restriction to the complex coefficients of a polynomial. A corrected statement of their result is given in Theorem 1.4 (some slight errors involving the powers of $t$ were present in the original statement of the result in [3]).

Theorem 1.4. Let $P(z)=\sum_{i=0}^{n} a_{j} z^{j}$ be a polynomial of degree $n$ with complex coefficients, where $\operatorname{Re}\left(a_{j}\right)=\alpha_{j}$ and $\operatorname{Im}\left(a_{j}\right)=\beta_{j}$ for $j=0,1,2, \ldots, n$, satisfying

$$
\begin{gathered}
\alpha_{0} \leq t^{2} \alpha_{2} \leq t^{4} \alpha_{4} \leq \cdots \leq t^{2 k} \alpha_{2 k} \geq t^{2 k+2} \alpha_{2 k+2} \geq \cdots \geq t^{2\lfloor n / 2\rfloor} \alpha_{2\lfloor n / 2\rfloor}, \\
\alpha_{1} \leq t^{2} \alpha_{3} \leq t^{4} \alpha_{5} \leq \cdots \leq t^{2 \ell-2} \alpha_{2 \ell-1} \geq t^{2 \ell} \alpha_{2 \ell+1} \geq \cdots \geq t^{2\lfloor n / 2\rfloor} \alpha_{2\lfloor(n+1) / 2\rfloor-1}, \\
\beta_{0} \leq t^{2} \beta_{2} \leq t^{4} \beta_{4} \leq \cdots \leq t^{2 s} \beta_{2 s} \geq t^{2 s+2} \beta_{2 s+2} \geq \cdots \geq t^{\lfloor n / 2\rfloor} \beta_{2\lfloor n / 2\rfloor}, \text { and } \\
\beta_{1} \leq t^{2} \beta_{3} \leq t^{4} \beta_{5} \leq \cdots \leq t^{2 q-2} \beta_{2 q-1} \geq t^{2 q} \beta_{2 q+1} \geq \cdots \geq t^{2\lfloor n / 2\rfloor} \beta_{2\lfloor(n+1) / 2\rfloor-1} \\
\text { for some } k, \ell, s, q \text { in }\{0,1, \ldots,\lfloor n / 2\rfloor\} . \text { Then all the zeros of } P \text { lie in } R_{1} \leq \\
|z| \leq R_{2} \text {, where } R_{1}=\min \left\{\frac{t\left|a_{0}\right|}{M_{1}}, t\right\}, R_{2}=\max \left\{\frac{M_{2}}{\left|a_{n}\right|}, \frac{1}{t}\right\}, \text { and } \\
M_{1}=-\left(\alpha_{0}+\beta_{0}\right)+\left(\left|\alpha_{1}\right|+\left|\beta_{1}\right|\right) t-\left(\alpha_{1}+\beta_{1}\right) t \\
\quad+2\left[\alpha_{2 k} t^{2 k}+2_{2 \ell-1} t^{2 \ell-1}+\beta_{2 s} t^{2 s}+\beta_{2 q-1} t^{2 q-1}\right]-\left(\alpha_{n-1}+\beta_{n-1}\right) t^{n-1} \\
\quad-\left(\alpha_{n}+\beta_{n}\right) t^{n}+\left(\left|\alpha_{n-1}\right|+\left|\beta_{n-1}\right|\right) t^{n-1}+\left(\left|\alpha_{n}\right|+\left|\beta_{n}\right|\right) t^{n}, \\
M_{2}=\left(\left|a_{0}\right| t^{4}-\left(\alpha_{0}+\beta_{0}\right)\right) t^{n-1}+\left(\left|a_{1}\right| t^{4}-\left(\alpha_{1}+\beta_{1}\right)\right) t^{n-2}
\end{gathered}
$$


AN ENESTRÖM-KAKEYA THEOREM FOR NEW CLASSES OF POLYNOMIALS 105

$$
\begin{aligned}
& +\left(t^{4}+1\right)\left(\alpha_{2 k} t^{n-1-2 k}+\alpha_{2 \ell-1} t^{n-2 \ell}+\beta_{2 s} t^{n-1-2 s}+\beta_{2 q-1} t^{n-2 q}\right) \\
& +\left(\left|a_{n-1}\right|-\left(\alpha_{n-1}+\beta_{n-1}\right) t^{4}\right)-\left(\alpha_{n}+\beta_{n}\right) t^{3} \\
& +\left(t^{4}-1\right)\left(\sum_{j=0, j \text { even }}^{2 k-2} \alpha_{j} t^{n-1-j}+\sum_{j=1, j \text { odd }}^{2 \ell-3} \alpha_{j} t^{n-1-j}\right. \\
& \left.+\sum_{j=0, j \text { even }}^{2 s-2} \beta_{j} t^{n-1-j}+\sum_{j=1, j \text { even }}^{2 q-3} \beta_{j} t^{n-1-j}\right) \\
& +\left(1-t^{4}\right)\left(\sum_{j=2 k+2, j \text { even }}^{2\lfloor n / 2\rfloor} \alpha_{j} t^{n-1-j}+\sum_{j\lfloor(n+1) / 2\rfloor-1} \alpha_{j} t^{n-1-j}\right. \\
& \left.+\sum_{j=2 s+2, j}^{j\lfloor n / 2\rfloor} \beta_{j} t^{n-1-j}+\sum_{j=2 q+1, j} \text { odd odd } \beta_{j} t^{n-1-j}\right)
\end{aligned}
$$

Notice the use of the parameters $2\lfloor n / 2\rfloor$ and $2\lfloor(n+1) / 2\rfloor-1$ in Theorem 1.4. Regardless of the parity of natural number $n$, the largest even natural number less than or equal to $n$ is $2\lfloor n / 2\rfloor$ and the largest odd natural number less than or equal to $n$ is $2\lfloor(n+1) / 2\rfloor-1$. In fact, $2\lfloor n / 2\rfloor=2\lceil(n-1) / 2\rceil$ and $2\lfloor(n+1) / 2\rfloor-1=2\lceil(n-2) / 2\rceil+1$. One can more generally show that the largest integer $N$ less than or equal to $n$ which is congruent to $k$ modulo $m$ is $N=m\lceil(n-m-k+1) / m\rceil+k$.

\section{Location of zeros results}

Inspired by the hypotheses of Theorem 1.4, we now present results for the class of polynomials which satisfy the monotonicity $t$ condition with a reversal as considered in Theorem 1.3, but as concerns the coefficients with indices the same modulo $m$. For example, if $m=2$, then we apply the condition to all coefficients with indices congruent to $0(\bmod 2)$ and $1(\bmod 2)$; that is, the condition is satisfied by the even indexed and odd indexed coefficients considered separately (as in Theorem 1.4). We consider polynomials satisfying this condition on the real part and imaginary part of the coefficients in our main result.

Theorem 2.1. Let $P(z)=\sum_{j=0}^{n} a_{j} z^{j}$ be a polynomial of degree $n$ with $\operatorname{Re}\left(a_{j}\right)=\alpha_{j}$ and $\operatorname{Im}\left(a_{j}\right)=\beta_{j}$, for some $m \in \mathbb{N}$ with $m<n$, and certain positive $t$,

$$
\begin{gathered}
\alpha_{k} \leq \alpha_{k+m} t^{m} \leq \alpha_{k+2 m} t^{2 m} \leq \cdots \leq \alpha_{r_{k}} t^{m\left\lfloor r_{k} / m\right\rfloor} \geq \alpha_{r_{k}+m} t^{m\left\lfloor r_{k} / m\right\rfloor+m} \geq \cdots \\
\geq \alpha_{m\lceil(n-m-k+1) / m\rceil+k} t^{m\lceil(n-m-k+1) / m\rceil}
\end{gathered}
$$


and

$$
\begin{gathered}
\beta_{k} \leq \beta_{k+m} t^{m} \leq \beta_{k+2 m} t^{2 m} \leq \cdots \leq \beta_{s_{k}} t^{m\left\lfloor s_{k} / m\right\rfloor} \geq \beta_{s_{k}+m} t^{m\left\lfloor s_{k} / m\right\rfloor+m} \geq \cdots \\
\geq \beta_{m\lceil(n-m-k+1) / m\rceil+k} t^{m\lceil(n-m-k+1) / m\rceil}
\end{gathered}
$$

for $k \in\{0,1, \ldots, m-1\}, r_{k} \equiv k(\bmod m), 0 \leq r_{k} \leq n, s_{k} \equiv k(\bmod m)$, and $0 \leq s_{k} \leq n$. Then all the zeros of $P$ lie in $R_{1} \leq|z| \leq R_{2}$, where

$$
R_{1}=\min \left\{\frac{t\left|a_{0}\right|}{M_{1}}, t\right\} \text { and } R_{2}=\max \left\{\frac{M_{2}}{\left|a_{n}\right|}, \frac{1}{t}\right\} \text {. }
$$

Here

$$
\begin{aligned}
M_{1}= & -\left(\alpha_{0}+\beta_{0}\right)+\sum_{k=1}^{m-1}\left(\left|a_{k}\right|-\left(\alpha_{k}+\beta_{k}\right)\right) t^{k}+2 \sum_{k=0}^{m-1}\left(\alpha_{r_{k}} t^{r_{k}}+\beta_{s_{k}} t^{s_{k}}\right) \\
& +\sum_{k=n-m+1}^{n}\left(\left|a_{k}\right|-\left(\alpha_{k}+\beta_{k}\right)\right) t^{k}
\end{aligned}
$$

and

$$
\begin{aligned}
& M_{2}=\sum_{k=0}^{m-1}\left(\left|a_{k}\right| t^{2 m}-\left(\alpha_{k}+\beta_{k}\right)\right) t^{n-1-k} \\
& +\left(t^{2 m}+1\right) \sum_{k=0}^{m-1}\left(\alpha_{r_{k}} t^{n-1-r_{k}}+\beta_{s_{k}} t^{n-1-s_{k}}\right) \\
& +\sum_{k=n-m+1}^{n-1}\left(\left|a_{k}\right|-\left(\alpha_{k}+\beta_{k}\right) t^{2 m}\right) t^{n-1-k}-\left(\alpha_{n}+\beta_{n}\right) t^{2 m-1} \\
& +\left(t^{2 m}-1\right) \sum_{k=0}^{m-1}\left(\sum_{\ell=1}^{\left(r_{k}-k\right) / m-1} \alpha_{k+\ell m} t^{n-1-k-\ell m}\right. \\
& \left.+\sum_{\ell=1}^{\left(s_{k}-k\right) / m-1} \beta_{k+\ell m} t^{n-1-k-\ell m}\right) \\
& +\left(1-t^{2 m}\right) \sum_{k=0}^{m-1}\left(\sum_{\ell=\left(r_{k}-k\right) / m+1}^{\lceil(n-m-k+1) / m\rceil-1} \alpha_{k+\ell m} t^{n-1-k-\ell m}\right. \\
& \left.+\sum_{\ell=\left(s_{k}-k\right) / m+1}^{\lceil(n-m-k+1) / m\rceil-1} \beta_{k+\ell m} t^{n-1-k-\ell m}\right) \text {. }
\end{aligned}
$$

Notice that when $m=2$, Theorem 2.1 reduces to Theorem 1.4. If the coefficients of $P$ are real, then Theorem 2.1 implies the following result. 
Corollary 2.1. Let $P(z)=\sum_{j=0}^{n} a_{j} z^{j}$ be a polynomial of degree $n$ with real coefficients where for some $m \in \mathbb{N}$ with $m<n$, and certain positive $t$,

$$
\begin{gathered}
a_{k} \leq a_{k+m} t^{m} \leq a_{k+2 m} t^{2 m} \leq \cdots \leq a_{r_{k}} t^{m\left\lfloor r_{k} / m\right\rfloor} \geq a_{r_{k}+m} t^{m\left\lfloor r_{k} / m\right\rfloor+m} \geq \cdots \\
\geq a_{m\lceil(n-m-k+1) / m\rceil+k} t^{m\lceil(n-m-k+1) / m\rceil}
\end{gathered}
$$

for $k \in\{0,1, \ldots, m-1\}, r_{k} \equiv k(\bmod m)$, and $0 \leq r_{k} \leq n$. Then all the zeros of $P$ lie in $R_{1} \leq|z| \leq R_{2}$, where

$$
R_{1}=\min \left\{\frac{t\left|a_{0}\right|}{M_{1}}, t\right\} \text { and } R_{2}=\max \left\{\frac{M_{2}}{\left|a_{n}\right|}, \frac{1}{t}\right\} \text {. }
$$

Here,

$$
M_{1}=-a_{0}+\sum_{k=1}^{m-1}\left(\left|a_{k}\right|-a_{k}\right) t^{k}+2 \sum_{k=0}^{m-1} a_{r_{k}} t^{r_{k}}+\sum_{k=n-m+1}^{n}\left(\left|a_{k}\right|-a_{k}\right) t^{k}
$$

and

$$
\begin{aligned}
M_{2}= & \sum_{k=0}^{m-1}\left\{\left(\left|a_{k}\right| t^{2 m}-a_{k}\right) t^{n-1-k}+\left(t^{2 m}+1\right) a_{r_{k}} t^{n-1-r_{k}}\right. \\
& +\left(t^{2 m}-1\right)\left(\sum_{\ell=1}^{\left(r_{k}-k\right) / m-1} a_{k+\ell m} t^{n-1-k-\ell m}\right) \\
& \left.+\left(1-t^{2 m}\right)\left(\sum_{\ell=\left(r_{k}-k\right) / m+1}^{\lceil(n-m-k+1) / m\rceil-1} a_{k+\ell m} t^{n-1-k-\ell m}\right)\right\} \\
& +\sum_{k=n-m+1}^{n-1}\left(\left|a_{k}\right|-a_{k} t^{2 m}\right) t^{n-1-k}-a_{n} t^{2 m-1} .
\end{aligned}
$$

In addition, if $t=1$, then Corollary 2.1 reduces to the following corollary.

Corollary 2.2. Let $P(z)=\sum_{j=0}^{n} a_{j} z^{j}$ be a polynomial of degree $n$ with real coefficients, where for some $m \in \mathbb{N}$ with $m<n$,

$$
a_{k} \leq a_{k+m} \leq a_{k+2 m} \leq \cdots \leq a_{r_{k}} \geq a_{r_{k}+m} \geq \cdots \geq a_{m\lceil(n-m-k+1) / m\rceil+k}
$$

for $k \in\{0,1, \ldots, m-1\}, r_{k} \equiv k(\bmod m)$, and $0 \leq r_{k} \leq n$. Then all the zeros of $P$ lie in $R_{1} \leq|z| \leq R_{2}$, where

$$
R_{1}=\min \left\{\frac{\left|a_{0}\right|}{M_{1}}, 1\right\} \text { and } R_{2}=\max \left\{\frac{M_{2}}{\left|a_{n}\right|}, 1\right\} .
$$

Here,

$$
M_{1}=-a_{0}+\sum_{k=1}^{m-1}\left(\left|a_{k}\right|-a_{k}\right)+2 \sum_{k=0}^{m-1} a_{r_{k}}+\sum_{k=n-m+1}^{n}\left(\left|a_{k}\right|-a_{k}\right),
$$




$$
M_{2}=\sum_{k=0}^{m-1}\left\{\left(\left|a_{k}\right|-a_{k}\right)+2 a_{r_{k}}\right)+\sum_{k=n-m+1}^{n-1}\left(\left|a_{k}\right|-a_{k}\right)-a_{n} .
$$

\section{Number of zeros results}

With the bounds $M_{1}$ and $M_{2}$ established above, we can easily prove results concerning the number of zeros in a disk for the classes of polynomials addressed in the previous section. For a list of several such previous results, see the introductory section of [9].

Theorem 3.1. Let $P(z)=\sum_{j=0}^{n} a_{j} z^{j}$ be a polynomial of degree $n$ with $\operatorname{Re}\left(a_{j}\right)=\alpha_{j}, \operatorname{Im}\left(a_{j}\right)=\beta_{j}$, and $a_{0} \neq 0$. Suppose the coefficients of $P$ satisfy the hypotheses of Theorem 2.1. Then, for $0<\delta<1$, the number of zeros of $P$ in the disk $|z| \leq \delta t$ is less than

$$
\frac{1}{\log 1 / \delta} \log \frac{M}{\left|t^{m} a_{0}\right|}
$$

where

$$
\begin{aligned}
M= & \sum_{k=0}^{m-1}\left(\left|a_{k}\right|-\left(\alpha_{k}+\beta_{k}\right)\right) t^{k+m}+2 \sum_{k=0}^{m-1}\left(\alpha_{r_{k}} t^{r_{k}+m}+\beta_{s_{k}} t^{s_{k}+m}\right) \\
& +\sum_{k=n-m+1}^{n}\left(\left|a_{k}\right|-\left(\alpha_{k}+\beta_{k}\right)\right) t^{k+m}
\end{aligned}
$$

With $m=2$ in Theorem 3.1 we get, as a corollary, Theorem 2.3 of [10].

We can extract a number of corollaries from Theorem 3.1. In particular, if $P$ has real coefficients, then with $t=1$ we have the following corollary.

Corollary 3.1. Let $P(z)=\sum_{j=0}^{n} a_{j} z^{j}$ be a polynomial of degree $n$ with real coefficients, where $a_{0} \neq 0$. Suppose the coefficients of $P$ satisfy the hypotheses of Corollary 2.2. Then, for $0<\delta<1$, the number of zeros of $P$ in the disk $|z| \leq \delta$ is less than

$$
\frac{1}{\log 1 / \delta} \log \frac{M}{\left|a_{0}\right|},
$$

where

$$
M=\sum_{k=0}^{m-1}\left(\left|a_{k}\right|-a_{k}\right)+2 \sum_{k=0}^{m-1} a_{r_{k}}+\sum_{k=n-m+1}^{n}\left(\left|a_{k}\right|-a_{k}\right) .
$$

As an example, consider the polynomial $P(z)=\left(3 z^{2}+10 z+1\right)\left(z^{6}+\right.$ $\left.z^{3}+1\right)=1+10 z+3 z^{2}+z^{3}+10 z^{4}+3 z^{5}+z^{6}+10 z^{7}+3 z^{8}$. P has the roots $(-5 \pm \sqrt{22}) / 3,1^{1 / 9}, 1^{2 / 9}, 1^{4 / 9}, 1^{5 / 9}, 1^{7 / 9}$, and $1^{8 / 9}$ (where we use the principal branch of the 9 th root function so that $1^{1 / 9}=\exp (2 \pi i / 9)$; notice that $(-5+\sqrt{22}) / 3 \approx-0.103,(-5-\sqrt{22}) / 3 \approx-3.230$, and that each of the 
remaining roots has modulus 1$)$. With $a_{0}=a_{3}=a_{6}=1, a_{1}=a_{4}=a_{7}=10$, $a_{2}=a_{5}=a_{8}=3, r_{0}=6, r_{1}=7$, and $r_{2}=8$, we see that Corollary 3.1 applies to $P$ with $m=3$. We find that $M=2\left(a_{6}+a_{7}+a_{8}\right)=2(1+10+3)=$ 28. With $\delta=0.15$, Corollary 3.1 implies that the number of zeros of $P$ in $|z| \leq \delta$ is less than

$$
\frac{1}{\log 1 / \delta} \log \frac{M}{\left|a_{0}\right|}=\frac{1}{\log 1 / 0.15} \log 28 \approx 1.76,
$$

which implies that $P$ has at most one zero in $|z| \leq 0.15$, namely $(-5+\sqrt{22}) / 3$. In fact, $P$ has exactly one zero in $|z| \leq 0.15$, and so Corollary 3.1 is sharp for this example.

\section{Proofs of results}

We need a lemma which appears in Titchmarsh's book [13] (see page 171 of the second edition).

Lemma 4.1. Let $F(z)$ be analytic in $|z| \leq R$. Let $|F(z)| \leq M$ in the disk $|z| \leq R$ and suppose $F(0) \neq 0$. Then, for $0<\delta<1$, the number of zeros of $F$ in the disk $|z| \leq \delta R$ is less than

$$
\frac{1}{\log 1 / \delta} \log \frac{M}{|F(0)|} .
$$

Proof of Theorem 2.1. Define $G(z)=\left(t^{m}-z^{m}\right) P(z)$. Then

$$
\begin{aligned}
G(z)= & \left(t^{m}-z^{m}\right) \sum_{j=0}^{n} a_{j} z^{j}=\sum_{j=0}^{n} a_{j} t^{m} z^{j}-\sum_{j=0}^{n} a_{j} z^{j+m} \\
= & \sum_{k=0}^{m-1}\left(a_{k} t^{m} z^{k}+a_{k+m} t^{m} z^{k+m}+a_{k+2 m} t^{m} z^{k+2 m}+\cdots\right. \\
& +a_{m\lceil(n-m-k+1) / m\rceil+k-m} t^{m} z^{m\lceil(n-m-k+1) / m\rceil+k-m} \\
& \left.+a_{m\lceil(n-m-k+1) / m\rceil+k} t^{m} z^{m\lceil(n-m-k+1) / m\rceil+k}\right) \\
& -\sum_{k=0}^{m-1}\left(a_{k} z^{k+m}+a_{k+m} z^{k+2 m}+a_{k+2 m} z^{k+3 m}+\cdots\right. \\
& +a_{m\lceil(n-m-k+1) / m\rceil+k-m} z^{m\lceil(n-m-k+1) / m\rceil+k} \\
& \left.+a_{m\lceil(n-m-k+1) / m\rceil+k} z^{m\lceil(n-m-k+1) / m\rceil+k+m}\right) \\
& =\sum_{k=0}^{m-1}\left(\alpha_{k} t^{m} z^{k}+\alpha_{k+m} t^{m} z^{k+m}+\alpha_{k+2 m} t^{m} z^{k+2 m}+\cdots\right. \\
& +\alpha_{m\lceil(n-m-k+1) / m\rceil+k-m} t^{m} z^{m\lceil(n-m-k+1) / m\rceil+k-m}
\end{aligned}
$$




$$
\begin{aligned}
& \left.+\alpha_{m\lceil(n-m-k+1) / m\rceil+k} t^{m} z^{m\lceil(n-m-k+1) / m\rceil+k}\right) \\
& +i \sum_{k=0}^{m-1}\left(\beta_{k} t^{m} z^{k}+\beta_{k+m} t^{m} z^{k+m}+\beta_{k+2 m} t^{m} z^{k+2 m}+\cdots\right. \\
& +\beta_{m\lceil(n-m-k+1) / m\rceil+k-m} t^{m} z^{m\lceil(n-m-k+1) / m\rceil+k-m} \\
& \left.+\beta_{m\lceil(n-m-k+1) / m\rceil+k} t^{m} z^{m\lceil(n-m-k+1) / m\rceil+k}\right) \\
& -\sum_{k=0}^{m-1}\left(\alpha_{k} z^{k+m}+\alpha_{k+m} z^{k+2 m}+\alpha_{k+2 m} z^{k+3 m}+\cdots\right. \\
& +\alpha_{m\lceil(n-m-k+1) / m\rceil+k-m} z^{m\lceil(n-m-k+1) / m\rceil+k} \\
& \left.+\alpha_{m\lceil(n-m-k+1) / m\rceil+k} z^{m\lceil(n-m-k+1) / m\rceil+k+m}\right) \\
& -i \sum_{k=0}^{m-1}\left(\beta_{k} z^{k+m}+\beta_{k+m} z^{k+2 m}+\beta_{k+2 m} z^{k+3 m}+\cdots\right. \\
& +\beta_{m\lceil(n-m-k+1) / m\rceil+k-m} z^{m\lceil(n-m-k+1) / m\rceil+k} \\
& \left.+\beta_{m\lceil(n-m-k+1) / m\rceil+k} z^{m\lceil(n-m-k+1) / m\rceil+k+m}\right) \\
& =\sum_{k=0}^{m-1} a_{k} t^{m} z^{k}+\sum_{k=0}^{m-1}\left\{\sum_{\ell=1}^{\left(r_{k}-k\right) / m}\left(\alpha_{k+\ell m} t^{m}-\alpha_{k+(\ell-1) m}\right) z^{k+\ell m}\right. \\
& \left.+\sum_{\ell=\left(r_{k}-k\right) / m+1}^{\lceil(n-m-k+1) / m\rceil}\left(\alpha_{k+\ell m} t^{m}-\alpha_{k+(\ell-1) m}\right) z^{k+\ell m}\right\} \\
& -\sum_{k=n-m+1}^{n} a_{k} z^{k+m} \\
& +i \sum_{k=0}^{m-1}\left\{\sum_{\ell=1}^{\left(s_{k}-k\right) / m}\left(\beta_{k+\ell m} t^{m}-\beta_{k+(\ell-1) m}\right) z^{k+\ell m}\right. \\
& \left.+\sum_{\ell=\left(s_{k}-k\right) / m+1}^{\lceil(n-m-k+1) / m\rceil}\left(\beta_{k+\ell m} t^{m}-\beta_{k+(\ell-1) m}\right) z^{k+\ell m}\right\} \\
& =a_{0} t^{m}+G_{1}(z) \text {. }
\end{aligned}
$$

On $|z|=t$, we have

$$
\left|G_{1}(z)\right| \leq \sum_{k=1}^{m-1}\left|a_{k}\right| t^{k+m}+\sum_{k=0}^{m-1}\left\{\sum_{\ell=1}^{\left(r_{k}-k\right) / m}\left|\alpha_{k+\ell m} t^{m}-\alpha_{k+(\ell-1) m}\right| t^{k+\ell m}\right.
$$


AN ENESTRÖM-KAKEYA THEOREM FOR NEW CLASSES OF POLYNOMIALS 111

$$
\begin{aligned}
& \left.+\sum_{\ell=\left(r_{k}-k\right) / m+1}^{\lceil(n-m-k+1) / m\rceil}\left|\alpha_{k+\ell m} t^{m}-\alpha_{k+(\ell-1) m}\right| t^{k+\ell m}\right\} \\
& +\sum_{k=n-m+1}^{n}\left|a_{k}\right| t^{k+m} \\
& +\sum_{k=0}^{m-1}\left\{\sum_{\ell=1}^{\left(s_{k}-k\right) / m}\left|\beta_{k+\ell m} t^{m}-\beta_{k+(\ell-1) m}\right| t^{k+\ell m}\right. \\
& \left.+\sum_{\ell=\left(s_{k}-k\right) / m+1}^{\lceil(n-m-k+1) / m\rceil}\left|\beta_{k+\ell m} t^{m}-\beta_{k+(\ell-1) m}\right| t^{k+\ell m}\right\} \\
& =\sum_{k=1}^{m-1}\left|a_{k}\right| t^{k+m}+\sum_{k=0}^{m-1}\left\{\sum_{\ell=1}^{\left(r_{k}-k\right) / m}\left(\alpha_{k+\ell m} t^{m}-\alpha_{k+(\ell-1) m}\right) t^{k+\ell m}\right. \\
& \left.+\sum_{\ell=\left(r_{k}-k\right) / m+1}^{\lceil(n-m-k+1) / m\rceil}\left(\alpha_{k+(\ell-1) m}-\alpha_{k+\ell m} t^{m}\right) t^{k+\ell m}\right\} \\
& +\sum_{k=0}^{m-1}\left\{\sum_{\ell=1}^{\left(s_{k}-k\right) / m}\left(\beta_{k+\ell m} t^{m}-\beta_{k+(\ell-1) m}\right) t^{k+\ell m}\right. \\
& \left.+\sum_{\ell=\left(s_{k}-k\right) / m+1}^{\lceil(n-m-k+1) / m\rceil}\left(\beta_{k+(\ell-1) m}-\beta_{k+\ell m} t^{m}\right) t^{k+\ell m}\right\} \\
& +\sum_{k=n-m+1}^{n}\left|a_{k}\right| t^{k+m} \\
& =\sum_{k=1}^{m-1}\left|a_{k}\right| t^{k+m}+\sum_{k=0}^{m-1}\left\{-\alpha_{k} t^{m+k}+2 \alpha_{r_{k}} t^{r_{k}+m}\right. \\
& \left.-\alpha_{k+m\lceil(n-m-k+1) / m\rceil} t^{k+m+m\lceil(n-m-k+1) / m\rceil}\right\} \\
& +\sum_{k=0}^{m-1}\left\{-\beta_{k} t^{m+k}+2 \beta_{s_{k}} t^{s_{k}+m}\right. \\
& \left.-\beta_{k+m\lceil(n-m-k+1) / m\rceil} t^{k+m+m\lceil(n-m-k+1) / m\rceil}\right\} \\
& +\sum_{k=n-m+1}^{n}\left|a_{k}\right| t^{k+m}
\end{aligned}
$$




$$
\begin{aligned}
= & -\alpha_{0} t^{m}-\beta_{0} t^{m}+\sum_{k=1}^{m-1}\left(\left|a_{k}\right|-\left(\alpha_{k}+\beta_{k}\right)\right) t^{k+m} \\
& +2 \sum_{k=0}^{m-1}\left(\alpha_{r_{k}} t^{r_{k}+m}+\beta_{s_{k}} t^{s_{k}+m}\right) \\
& +\sum_{k=n-m+1}^{n}\left(\left|a_{k}\right|-\left(\alpha_{k}+\beta_{k}\right)\right) t^{k+m} \\
= & t^{m} M_{1} .
\end{aligned}
$$

Applying Schwarz's Lemma (see page 168 of [13]) to $G_{1}(z)$, we get

$$
\left|G_{1}(z)\right| \leq \frac{t^{m} M_{1}|z|}{t}=t^{m-1} M_{1}|z| \text { for }|z| \leq t
$$

This implies

$$
\begin{aligned}
|G(z)| & =\left|\left(t^{m}-z^{m}\right) P(z)\right|=\left|a_{0} t^{m}+G_{1}(z)\right| \\
& \geq t^{m}\left|a_{0}\right|-\left|G_{1}(z)\right| \geq t^{m}\left|a_{0}\right|-t^{m-1} M_{1}|z| \text { for }|z| \leq t .
\end{aligned}
$$

Hence, if $|z|<R_{1}=\min \left\{t\left|a_{0}\right| / M_{1}, t\right\}$, then $G(z) \neq 0$ and so $P(z) \neq 0$.

Next we take $G(z)=\left(t^{m}-z^{m}\right) P(z)=G_{2}(z)-a_{n} z^{n+m}$ and so

$$
\begin{aligned}
& G_{2}(z)=\sum_{k=0}^{m-1} a_{k} t^{m} z^{k}+\sum_{k=0}^{m-1}\left\{\sum_{\ell=1}^{\left(r_{k}-k\right) / m}\left(\alpha_{k+\ell m} t^{m}-\alpha_{k+(\ell-1) m}\right) z^{k+\ell m}\right. \\
& \left.+\sum_{\ell=\left(r_{k}-k\right) / m+1}^{\lceil(n-m-k+1) / m\rceil}\left(\alpha_{k+\ell m} t^{m}-\alpha_{k+(\ell-1) m}\right) z^{k+\ell m}\right\} \\
& -\sum_{k=n-m+1}^{n-1} a_{k} z^{k+m} \\
& +i \sum_{k=0}^{m-1}\left\{\sum_{\ell=1}^{\left(s_{k}-k\right) / m}\left(\beta_{k+\ell m} t^{m}-\beta_{k+(\ell-1) m}\right) z^{k+\ell m}\right. \\
& \left.+\sum_{\ell=\left(s_{k}-k\right) / m+1}^{\lceil(n-m-k+1) / m\rceil}\left(\beta_{k+\ell m} t^{m}-\beta_{k+(\ell-1) m}\right) z^{k+\ell m}\right\} \text {. }
\end{aligned}
$$

Then

$$
z^{n+m-1} G_{2}\left(\frac{1}{z}\right)=\sum_{k=0}^{m-1} a_{k} t^{m} z^{n+m-1-k}
$$


AN ENESTRÖM-KAKEYA THEOREM FOR NEW CLASSES OF POLYNOMIALS 113

$$
\begin{aligned}
& +\sum_{k=0}^{m-1}\left\{\sum_{\ell=1}^{\left(r_{k}-k\right) / m}\left(\alpha_{k+\ell m} t^{m}-\alpha_{k+(\ell-1) m}\right) z^{n+m-1-k-\ell m}\right. \\
& \left.+\sum_{\ell=\left(r_{k}-k\right) / m+1}^{\lceil(n-m-k+1) / m\rceil}\left(\alpha_{k+\ell m} t^{m}-\alpha_{k+(\ell-1) m}\right) z^{n+m-1-k-\ell m}\right\} \\
& -\sum_{k=n-m+1}^{n-1} a_{k} z^{n-1-k} \\
& +i \sum_{k=0}^{m-1}\left\{\sum_{\ell=1}^{\left(s_{k}-k\right) / m}\left(\beta_{k+\ell m} t^{m}-\beta_{k+(\ell-1) m}\right) z^{n+m-1-k-\ell m}\right. \\
& \left.+\sum_{\ell=\left(s_{k}-k\right) / m+1}^{\lceil(n-m-k+1) / m\rceil}\left(\beta_{k+\ell m} t^{m}-\beta_{k+(\ell-1) m}\right) z^{n+m-1-k-\ell m}\right\} \text {. }
\end{aligned}
$$

For $|z|=t$ we have

$$
\begin{aligned}
& \left|z^{n+m-1} G_{2}\left(\frac{1}{z}\right)\right| \leq \sum_{k=0}^{m-1}\left|a_{k}\right| t^{n+2 m-1-k} \\
& +\sum_{k=0}^{m-1}\left\{\sum_{\ell=1}^{\left(r_{k}-k\right) / m}\left|\alpha_{k+\ell m} t^{m}-\alpha_{k+(\ell-1) m}\right| t^{n+m-1-k-\ell m}\right. \\
& \left.+\sum_{\ell=\left(r_{k}-k\right) / m+1}^{\lceil(n-m-k+1) / m\rceil}\left|\alpha_{k+\ell m} t^{m}-\alpha_{k+(\ell-1) m}\right| t^{n+m-1-k-\ell m}\right\} \\
& +\sum_{k=0}^{m-1}\left\{\sum_{\ell=1}^{\left(s_{k}-k\right) / m}\left|\beta_{k+\ell m} t^{m}-\beta_{k+(\ell-1) m}\right| t^{n+m-1-k-\ell m}\right. \\
& \left.+\sum_{\ell=\left(s_{k}-k\right) / m+1}^{\lceil(n-m-k+1) / m\rceil}\left|\beta_{k+\ell m} t^{m}-\beta_{k+(\ell-1) m}\right| t^{n+m-1-k-\ell m}\right\} \\
& +\sum_{k=n-m+1}^{n-1}\left|a_{k}\right| t^{n-1-k} \\
& =\sum_{k=0}^{m-1}\left\{\left(\left|a_{k}\right| t^{2 m}-\left(\alpha_{k}+\beta_{k}\right)\right) t^{n-1-k}+\left(t^{2 m}+1\right)\left(\alpha_{r_{k}} t^{n-1-r_{k}}\right.\right.
\end{aligned}
$$




$$
\begin{aligned}
& \left.+\beta_{s_{k}} t^{n-1-s_{k}}\right)+\left(t^{2 m}-1\right)\left(\sum_{\ell=1}^{\left(r_{k}-k\right) / m-1} \alpha_{k+\ell m} t^{n-1-k-\ell m}\right. \\
& \left.+\sum_{\ell=1}^{\left(s_{k}-k\right) / m-1} \beta_{k+\ell m} t^{n-1-k-\ell m}\right) \\
& +\left(1-t^{2 m}\right)\left(\begin{array}{c}
\lceil(n-m-k+1) / m\rceil-1 \\
\ell=\left(r_{k}-k\right) / m+1
\end{array}\right) \\
& \left.\begin{array}{l}
\lceil(n-m-k+1) / m\rceil-1 \\
\left.\beta_{k+\ell m} t^{n-1-k-\ell m}\right)
\end{array}\right\} \\
& +\sum_{k=\left(s_{k}-k\right) / m+1}^{n-1}\left(\left|a_{k}\right|-\left(\alpha_{k}+\beta_{k}\right) t^{2 m}\right] t^{n-1-k-\ell m}+ \\
& =M_{2} .
\end{aligned}
$$

Hence, by the Maximum Modulus Theorem,

$$
\left|z^{n+m-1} G_{2}\left(\frac{1}{z}\right)\right| \leq M_{2} \text { for }|z| \leq t,
$$

which implies that

$$
\left|G_{2}(z)\right| \leq M_{2}|z|^{n+m-1} \text { for }|z| \geq \frac{1}{t}
$$

Therefore, for $|z| \geq 1 / t$ we have

$$
\begin{aligned}
|G(z)| & =\left|-a_{n} z^{n+m}+G_{2}(z)\right| \geq\left|a_{n}\right||z|^{n+m}-M_{2}|z|^{n+m-1} \\
& =|z|^{n+m-1}\left(\left|a_{n}\right||z|-M_{2}\right) .
\end{aligned}
$$

So, if $|z|>R_{2}=\max \left\{\frac{M_{2}}{\left|a_{n}\right|}, \frac{1}{t}\right\}$, then $G(z) \neq 0$ and hence $P(z) \neq 0$. The proof is complete.

Proof of Theorem 3.1. As in the proof of Theorem 2.1, for $|z|=t$ we have

$$
\begin{aligned}
|G(z)|= & \left|\left(t^{m}-z^{m}\right) P(z)\right|=\left|a_{0} t^{m}+G_{1}(z)\right| \leq\left|a_{0}\right| t^{m}+\left|G_{1}(z)\right| \\
\leq & \sum_{k=0}^{m-1}\left(\left|a_{k}\right|-\left(\alpha_{k}+\beta_{k}\right)\right) t^{k+m}+2 \sum_{k=0}^{m-1}\left(\alpha_{r_{k}} t^{r_{k}+m}+\beta_{s_{k}} t^{s_{k}+m}\right) \\
& +\sum_{k=n-m+1}^{n}\left(\left|a_{k}\right|-\left(\alpha_{k}+\beta_{k}\right)\right) t^{k+m}=M .
\end{aligned}
$$

Now $G(z)$ is analytic in $|z| \leq t$, and $|G(z)| \leq M$ for $|z|=t$. So, by Lemma 4.1 and the Maximum Modulus Theorem, the number of zeros of $G$ (and 
hence of $P$ ) in $|z| \leq \delta t$ is less than

$$
\frac{1}{\log 1 / \delta} \log \frac{M}{|G(0)|}=\frac{1}{\log 1 / \delta} \log \frac{M}{\left|t^{m} a_{0}\right|} .
$$

\section{References}

[1] A. Aziz and Q. G. Mohammad, On the zeros of a certain class of polynomials and related analytic functions, J. Math. Anal. Appl. 75 (1980), 495-502.

[2] A. Aziz and B. A. Zargar, Some extensions of Eneström-Kakeya Theorem, Glas. Math. 31(51) (1996), 239-244.

[3] J. Cao and R. Gardner, Restrictions on the zeros of a polynomial as a consequence of conditions on the coefficients of even powers and odd powers of the variable, J. Comput. Appl. Math. 155 (2003), 153-163.

[4] G. Eneström, Härledning af en allmän formel för antalet pensionärer, som vid en godtyeklig tidpunkt förefinnas inom en sluten pensionslcassa, Övfers. Vetensk.-Akad. Fórhh. 50 (1893), 405-415.

[5] G. Eneström, Remarque sur un th'eorème relatif aux racines de l'equation $a_{n} x^{n}+$ $a_{n-1} x^{n-1}+\cdots+a_{1} x+a_{0}=0$ où tous les coefficientes a sont réel et positifs, Tôhoku Math. J. 18 (1920), 34-36.

[6] R. Gardner and N. K. Govil, On the location of the zeros of a polynomial, J. Approx. Theory 78 (1994), 286-292.

[7] R. Gardner and N. K. Govil, Some generalizations of the Eneström-Kakeya Theorem, Acta Math. Hungarica 74(1-2) (1997), 125-134.

[8] R. Gardner and N. K. Govil, The Enestrom-Kakeya Theorem and Some of Its Generalizations, in: Current Topics in Pure and Computational Complex Analysis (Springer Verlag), eds. S. Joshi, M. Dorff, and I. Lahiri, New Delhi, 2014, pp. 171-200.

[9] R. Gardner and B. Shields, The number of zeros of a polynomial in a disk, J. Class. Anal. 3(2) (2013), 167-176.

[10] R. Gardner and B. Shields, The number of zeros of a polynomial in a disk as a consequence of restrictions on the coefficients, Acta Comment. Univ. Tartu. Math. 19(2) (2015), 109-120.

[11] A. Joyal, G. Labelle, and Q. I. Rahman, On the location of zeros of polynomials, Canad. Math. Bull. 10(1) (1967), 53-63.

[12] S. Kakeya, On the limits of the roots of an algebraic equation with positive coefficients, Tôhoku Math. J. 2 (1912-1913), 140-142.

[13] E. C. Titchmarsh, The Theory of Functions, 2nd Ed., Oxford University Press, London, 1939.

School of Mathematics, University of Minnesota, Minneapolis, Minnesota 55455 , USA

E-mail address: frazi197@umn.edu

Department of Mathematics and Statistics, East Tennessee State UniverSity, Johnson City, Tennessee 37614-0663, USA

E-mail address: gardnerr@etsu.edu 